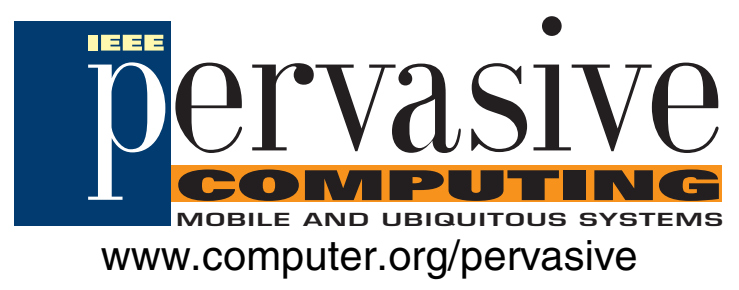

\title{
The Webkit Tangible User Interface: A Case Study of Iterative Prototyping
}

\author{
Mark Stringer, Jennifer A. Rode, Eleanor F. Toye, \\ Alan F. Blackwell, and Amanda R. Simpson
}

Vol. 4, No. 4

October-December 2005

This material is presented to ensure timely dissemination of scholarly and technical work. Copyright and all rights therein are retained by authors or by other copyright holders. All persons copying this information are expected to adhere to the terms and constraints invoked by each author's copyright. In most cases, these works may not be reposted without the explicit permission of the copyright holder.
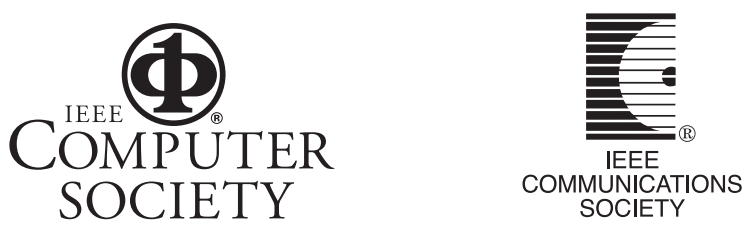

(C) 2005 IEEE. Personal use of this material is permitted. However, permission to reprint/republish this material for advertising or promotional purposes or for creating new collective works for resale or redistribution to servers or lists, or to reuse any copyrighted component of this work in other works must be obtained from the IEEE. 


\title{
The Webkit Tangible User Interface: A Case Study of Iterative Prototyping
}

\author{
Designs must account for social consequences—not just form factors and \\ other $\mathrm{HCl}$ concerns. In situ iterative prototyping and a new user-centered \\ design method can help.
}

\section{Mark Stringer \\ University of Sussex}

Jennifer A. Rode

University of California, Irvine

Eleanor F. Toye

Framfab UK

Alan F. Blackwell

University of Cambridge

Amanda R. Simpson

University of Central England

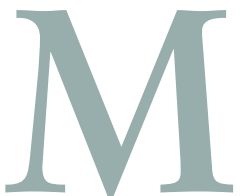

ark Weiser envisioned technologies that weave themselves into the fabric of everyday life such that they disappear. ${ }^{1}$ Although disappearing is due partly to miniaturization and clever manipulation of the technological artifacts themselves, more than physical size and the hiding of technology is at stake. We must integrate technology into the social fabric of everyday life to ensure its appropriateness in everyday activities. So, we must design with regard to not only the form factor and other traditional HCI concerns but also our prototype's social consequences. In situ iterative prototyping is critical to achieving these ends.

Here we describe our 18month iterative-design program to create Webkit, a tangible user interface (TUI) to help school children learn argumentation skills—an English National Curriculum requirement. The goal was to have Webkit disappear into the environment such that the teachers and students remained focused on the learning activity. However, the school children wouldn't be allowed to evaluate our prototypes unless we showed that the classroom time allocated to our research simultane- ously helped teach the curriculum. To achieve this, we developed a form of user-centered design called curriculum-focused design. ${ }^{2}$ When we used CFD, the curriculum and student needs motivated not just the final technology but also our design process.

Although the specifics of the educational setting might not be universally applicable, our strategy offers a case study of design practice. Frequently evaluating prototypes that increase in technological complexity, and conducting these evaluations in the environment in which the technology will ultimately be used, lets you effectively address contextual factors.

\section{The Webkit project}

Webkit was part of a project funded to explore how to use RFID technology to apply TUIs to the World Wide Web. Our technical objective was to explore TUIs' potential in a classroom of children aged 11 to 13 (years seven and eight in the English state school system) to enhance student interaction with the Web.

Researchers have discussed at length TUIs' potential as a rich, expressive means of input and have pointed out that TUIs lend themselves naturally to collaborative activities. ${ }^{3}$ (Also see the "Related Work" sidebar.) However, we were cautious in our approach, because some HCI research in educational settings has indicated that collaborative technologies can be gratuitous or 


\section{Related Work in Tangible Interfaces for Children}

$\mathrm{T}$ here's much interest in designing tangible user interfaces for children. ${ }^{1,2}$ Oren Zuckerman, Saeed Arida, and Mitchel Resnick have used TUls to teach abstract thinking skills to children, but they don't focus on argumentation skills. Their research presents two completed prototypes and the results from their qualitative studies, with little insight into the design process that allowed them to arrive at their design. Diana Africano and her colleagues have used our curriculum-focused design method, but they focus on their final design and evaluation, again with little insight into the design process. So, our work is novel as a case study of iterative TUI creation as an example of how to perform curriculum-focused design.

\section{REFERENCES}

1. O. Zuckerman, S. Arida, and M. Resnick, "Extending Tangible Interfaces for Education: Digital Montessori-Inspired Manipulatives," Proc. SIGCHI Human Factors Conf. in Computing Systems (CHI 05), ACM Press, 2005, pp. 859-868.

2. D. Africano at al., "Designing Tangible Interfaces for Children's Collaboration," $\mathrm{CHI}$ '04 Extended Abstracts on Human Factors in Computing Systems, ACM Press, 2004, pp. 853-868.

unhelpful for educational purposes. ${ }^{4-6}$ We therefore wished to apply principles of learner-centered design to focus on educational benefits for the student. ${ }^{7}$

Furthermore, the constraints of the English National Curriculum meant we couldn't apply established prototyping methods such as Allison Druin's cooperative inquiry. ${ }^{8}$ Druin followed a participatory design philosophy that elevates children to the role of full-fledged members of the design team. The children engage in contextual inquiry with other students and participate actively in prototype creation. In our more constrained and targeted context, the prototypes we tested in classrooms couldn't fail in such a way that they disrupted or derailed the lesson.

So, we conducted much of our work using reliable but low-tech prototypes, which had several advantages. Most important, this let us rapidly produce and test a variety of prototypes. So, we gained a better understanding of desirable interactions before considering how RFID technology could augment and support the activities.

We took advantage of having a former assistant head teacher with 10 years of experience as part of the research team.
The teacher acted as a facilitator, so we maintained the traditional power dynamic of the classroom while the students participated in the design process. We thus avoided some of the social awkwardness Druin claimed was produced by the egalitarian nature of her intergenerational design teams.

We invited the students to give written feedback using questionnaires and verbal feedback in sessions. The questionnaires asked the students about their interaction with the technology-what they liked and disliked-and let them express their opinions without any social pressures. We then used the students' feedback in the next iteration, along with the facilitator's opinion of how well we achieved the lesson objectives and the other researchers' observations of the students' reactions to the interface.

Over the course of the project, we produced 11 distinct prototypes. To ensure our educational goals were in concert with the teachers' goals, we began by developing a series of "no technology" prototypes. The CFD approach let us gain progressive feedback as we gradually introduced technology, beginning with printed Web pages, then Wizard of $\mathrm{Oz}$ style mock-ups, and finally fully robust technologies using RFID. (With the Wizard of Oz approach, a human carries out the processing and generates the interactions that the computer will eventually perform.) Iteratively evolving the design over a series of prototypes let us explore the minutiae of interaction possibilities and arrive at a form factor and functionalities for the design that achieved the teacher's educational goals.

\section{Initial prototypes: Iterations 1 through 4}

Our research began with an observation phase that involved minimal classroom intervention. An observer spent three weeks recording the students' normal school activities and interviewing teachers to identify which parts of the curriculum would benefit most from enhanced physical objects.

We also observed school children visiting the Science Museum in London, where they interact with a wider variety of tangible educational materials-some technically sophisticated, some low tech. Of particular interest was the Launch Pad, a basement area that contained numerous low-tech, hands-on demonstrations of physical and engineering concepts. One of the most popular was a grain pit where children could move grain around using grasping crane buckets, hand-turned conveyor belts, and an Archimedes screw. This room exhibited a higher level of engagement and excitement than those containing flat-panel computer displays.

\section{Iteration 1:}

\section{D conceptual prototypes}

Our ethnographic study identified various physical objects that we could enhance physically and electronically to structure and check logical arguments. We developed these through conceptualdesign workshops, constructing many exploratory 3D sketches from craft materials (see figure 1a). 
Figure 1. Initial Webkit prototypes: (a) three conceptual prototypes (iteration 1), (b) the selection prototype (iteration 3 ), and (c) on-the-floor prototypes (iterations 5 and 6).

\section{Iteration 2: Paper prototype}

To determine how children would research and construct a persuasive argument from Web resources, we ran a paper-only trial. We gave a small group of year-seven children, supervised by our researcher/teacher, Web page printouts relevant to a topic from their history class. We asked the students to use this information to construct a convincing argument.

The classroom observer recorded three one-hour sessions on video, which the entire design team later analyzed. In these sessions, the girls dominated the interaction-this wasn't surprising given that girls of this age have more advanced verbal skills than their male counterparts.

On the basis of our observations, we developed a preliminary model of an argument formation cycle (see figure 2). The cycle starts with a long period in which the students read, evaluate, and selectively highlight source pages. Then they group relevant pieces of evidence, name each group, and structure the argument. The final stage involves turning the argument structure into a linear form that the students can use to deliver a speech or write an essay.

Our approach was similar to Abigail Sellen and Richard Harper's discussion of the document life cycle. ${ }^{9}$ Just as they highlighted parts of the document-creation life cycle that were best supported by paper, we hoped to identify parts of the argument formation cycle that TUIs could best augment.

\section{Iterations 3 and 4: Evidence collection}

Our first trials of a $3 \mathrm{D}$ prototype

Figure 2. Our argument formation cycle, which we used to identify possible target areas for digital augmentation.

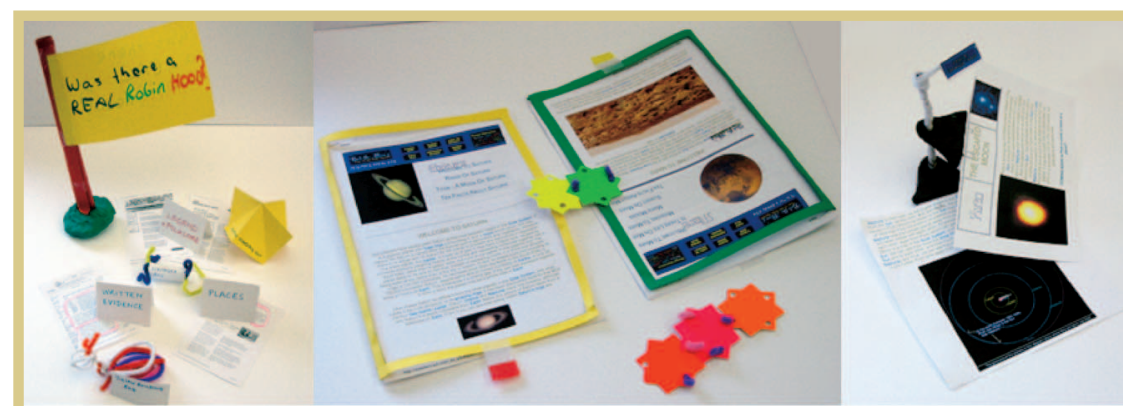

(a)

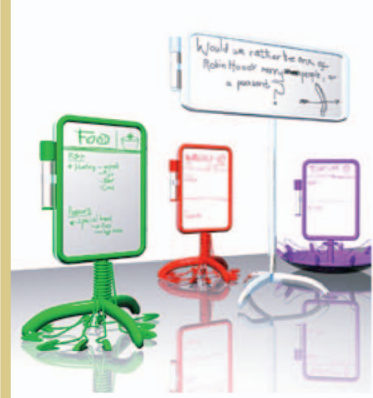

(b)

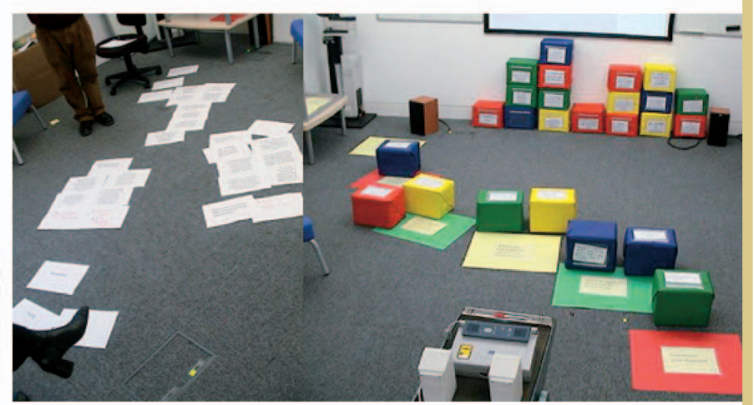

(c) focused on the argument formation cycle's selection, collection, and labeling phases. We gave year-seven children whiteboards on small stands (see figure 1b) on which to write a group heading and attach supporting evidence called statements. We intended to later have an RFID reader in the stand that could recognize RFID tags in the documents so that the system could identify the logical relationship between statements and collections of documents.

However, we observed two problematic use patterns:

- The students no longer collaborated as they had done with the piles of paper. Instead, each student adopted one stand, collecting evidence just on that topic.

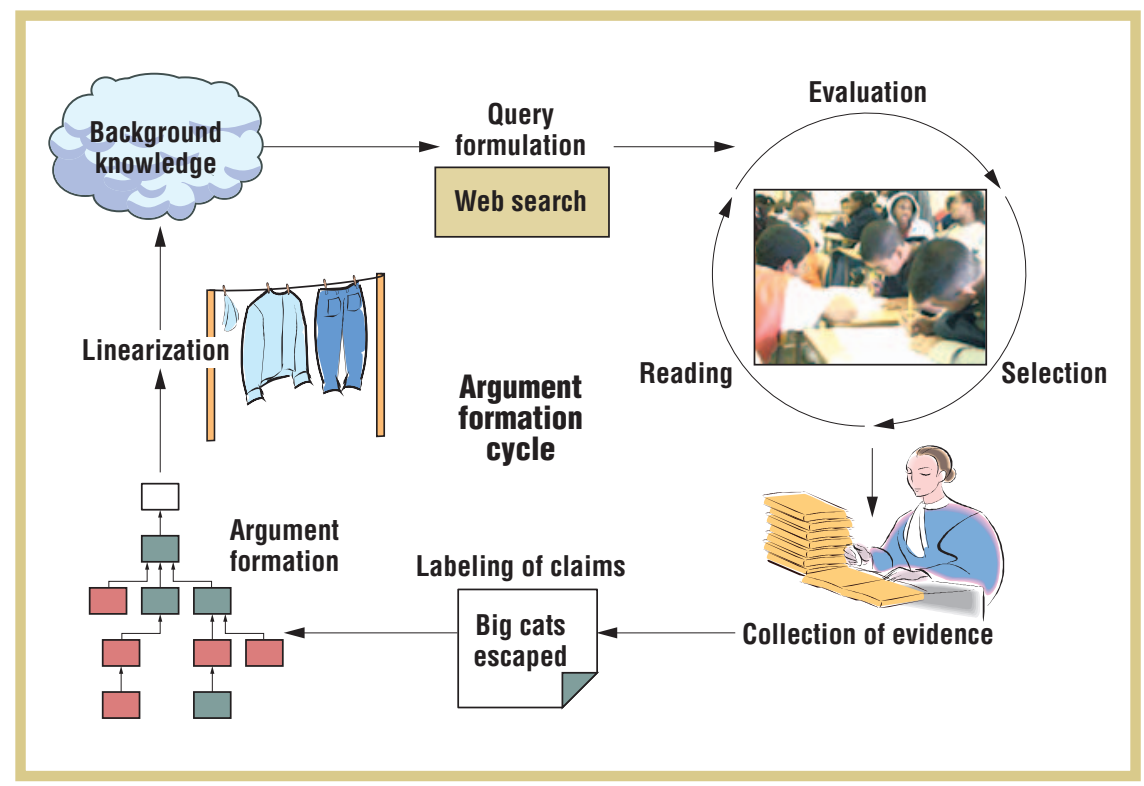


- The students transcribed evidence onto the whiteboard rather than attaching it to the clips. This further prohibited collaboration and would make the proposed RFID operation ineffective.

We addressed these issues in iteration 4 with two subtle design changes. We drastically reduced the writing area on the board to discourage transcription. We also ensured there were fewer stands than students, which encouraged collaboration. These nontechnical solutions were far cheaper to implement at this stage, before we had developed fully functional RFID prototypes.

\section{The Tangible User Interface: Iterations 5 through 11}

The paper prototype trials led us to discard assumptions we had taken from prior research in computer-supported collaborative argumentation. ${ }^{10}$ We originally expected the TUI to help the students understand the logical relations between points in an argument. However, the students already understood the logical implications; it was the argument's rhetorical structure that confused them. For example, they didn't know what to do with material found during their research that didn't support their side of the debate. This suggested a different role for the TUI.

The Greek technique of teaching rhetoric used a series of exercises known as the progymnasmata. ${ }^{11}$ These exercises broke down argument construction into a series of manageable steps to ensure debaters covered the necessary ground and used their research as effectively as possible. With help from the teacher, we updated three of these classical exercises:

- vituperation-a speech that opposes a person or idea,

- encomium-a speech that praises a person or idea, and
- thesis-a balanced argument dealing with points for and against an idea.

The students performed each exercise for a certain topic. We divided each exercise into five stages, each of which had a large card stating what the student should do—for example, "say something actions we saw in the Launch Pad during the ethnographic study. By iterating on the form factor, we moved from statement-holders that encouraged isolated work to ones that were more naturally collaborative. We were trying to improve discursive skills, so the students' discussion while creating their arguments was

\section{The paper prototype trials led us to discard}

\section{assumptions we had taken from prior research in}

\section{computer-supported collaborative}

\section{argumentation.}

good about Robin Hood"-during that stage of the argument. We called these cards the statement-holders. Students placed statements containing evidence found in their research on a suitable category to structure their argument. These statement-holders helped the students structure their arguments.

\section{Iteration 5: A low-tech prototype}

In iteration 4, the students spent too much time copying down statements from references. We wanted to encourage the students to think about the argument structure rather than just recall the material outright. So, our next iteration focused exclusively on the lesson rather than the form factor. We let students record statements online and then print them, eliminating transcription.

Although this approach showed promise, the printed statements and statement-holders were hard to distinguish from a distance and tended to overlap, presenting a legibility problem.

\section{Iteration 6: A medium-tech prototype}

We improved visibility by attaching the statements printed on a half sheet of paper to large, brightly colored boxes. This echoed the compelling, large inter- vital to the lesson. We also increased the statement-holders' size and improved the contrast by placing the black and white text on a colored background. Figure 1c shows the statement-holders flat on the floor with statement boxes placed on top of them; unused statements are stacked against the wall.

We added a GUI projected onto the wall to further improve visibility (see figure 3a). It showed a map of the argument that duplicated the TUI's layout (see figure 3b), and we updated it using a Wizard of $\mathrm{Oz}$ approach whenever a student arranged the statements. The students could print the GUI's argument map at the lesson's end, so they could work on it later or prepare a paper or speech based on the argument structure.

This large-scale prototype was highly engaging, with students enthusiastically building towers of related statements on relevant statement-holders. Unfortunately, the individual boxes' size meant that an RFID reader couldn't detect statements higher in the tower. This wasn't a problem in our Wizard of $\mathrm{Oz}$ simulation, which sufficiently showed the advantage of digitally visualizing the argument structure, but it would be a serious problem in a final high-tech implementation. Another problem with the towers was that the 
Figure 3. Introducing the technology: (a) a GUI that projected an argument map onto the wall, (b) a sample argument map, and (c) detailed argument points from a lesson on debating the scientific validity of ghosts.

statements chosen first were typically the most important, but they weren't as visible at the bottom of the pile. So, when students talked through the argument, they started in reverse order of importance by starting with the box on top.

\section{Iteration 7: A high-tech prototype}

To assess our design decisions' implications for technological implementation, we started building active prototypes that included RFID tags in the statement boxes and RFID readers in the statement-holders. This RFID technology didn't support the "collision detection" method by which a single reader can detect multiple ID tags, so we couldn't place two different statement boxes on a statement-holder at the same time. We redesigned the statement-holders so that each had a target on which the students could place a statement they wished to register. The students then had to remove the statement before registering another one, which enforced unnecessary turn-taking. This also meant that the registered statements' physical arrangement that had been registered didn't reflect the recorded system status.

This change to accommodate a specific version of the RFID reading technology disrupted the lesson, as did the performance of the prototype reader hardware: it identified the boxes' ID tags only about 50 percent of the time. For the first time, our prototype stalled the lesson, and the students lost interest in the activity.

The benefits of our multiple low-tech iterations became clear at this stage. We had developed and evaluated a wide range of TUI options that let us explore desirable interactions in the educational context with relatively little distraction from the limitations of electronic-prototyping cycles.
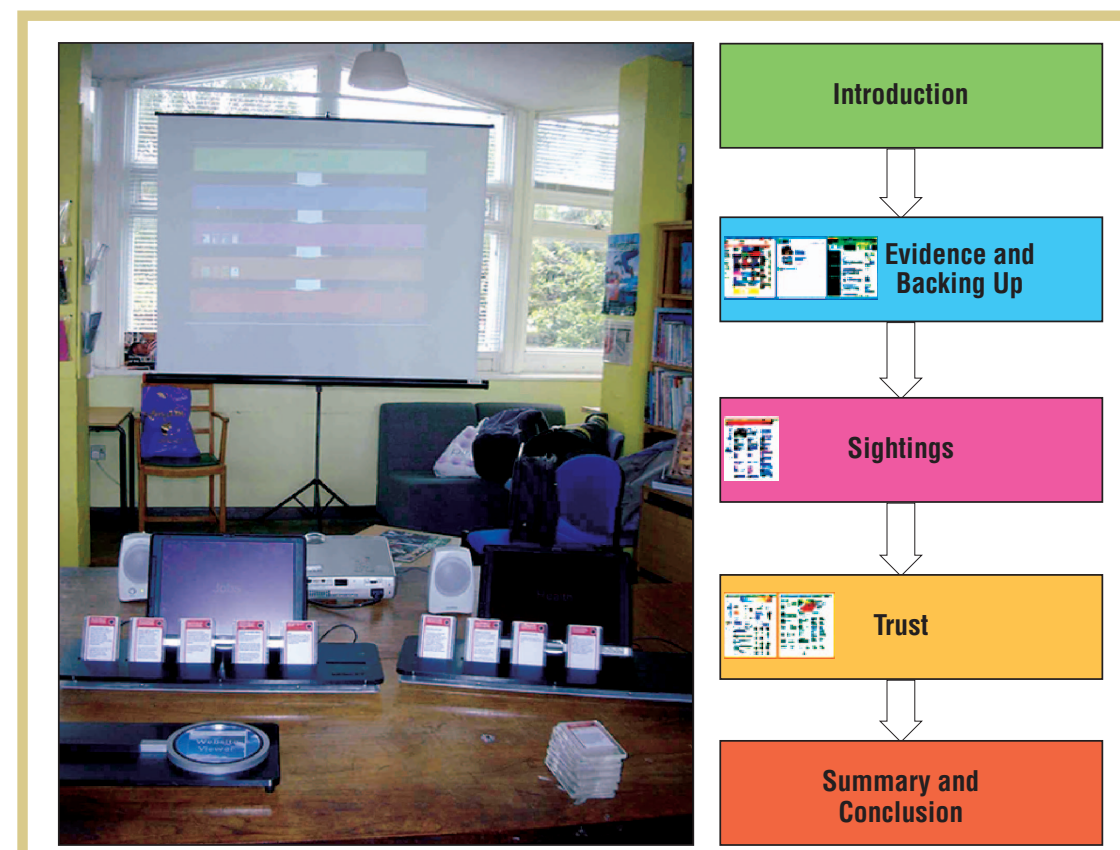

(a)

(b)

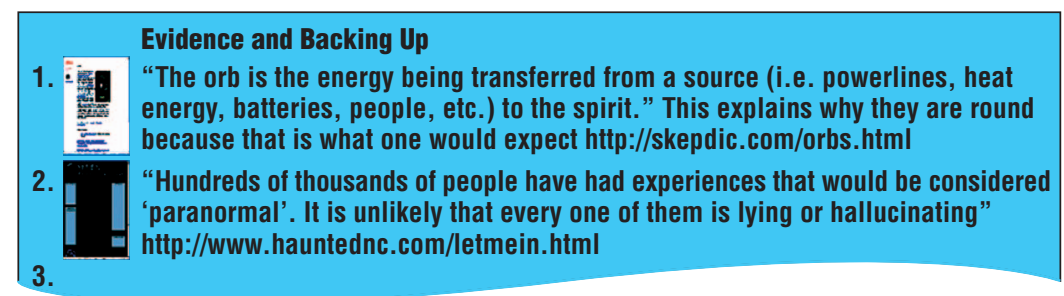

(c)

\section{Iterations 8 and 9: Exploring form factors}

Our iterative process let us explore various form factors including whether the large boxes' educational value justified the inconvenience of storing them and clearing the floor space. So, we created 3D design sketches examining alternative embodiments (iterations 8, 9 , and 10). Iteration 8 used statements on small flags, which students tended to twirl between their fingers, exacerbating the visualization problem. Iteration 9 used small boxes with the text displayed on three sides. Ultimately, working with the teachers, we determined that the boxes were more practical in the classroom and were similarly engaging. This form factor encouraged students to use the boxes like bricks, building walls with arbitrary order. It was this problem of arbitrary order we tried to address in iteration 10 , using slotted statement-holders.

\section{Iteration 10: Near-final, table-top version}

Taking into account our experiences from the preceding design iterations, we implemented and evaluated a table-top version of the application. This iteration represented our near-final form factor. The design let students explore evidence selection, argument construction, and argument presentation.

We followed through with our plan to place an RFID tag in each statement card and an RFID reader in each stand. Each statement-holder also contained an inductive circuit that lit up an LED light whenever the statement-holder was within an RFID-reading antenna's range. This prototype's RFID technology contained collision-detection software, so the students 
could place more than one statement card on the statement-holder at a time.

To help the students construct their arguments, we created statement-holders using statements produced in earlier stages of the argument formation cycle. We printed the statements on labels that we then attached to RFID-enhanced cards. The students could place these cards into the newly designed slots on the statement-holders. This addressed the problems encountered with stacked boxes, because it allowed left-to-right reading of the statement cards. As we had seen in previous trials, the most important statements tended to be put down first. We now saw the students arranging the statements so they read from left to right-putting the most important statements first so these were the first cards read when the students delivered their speeches.

After placing a card on the statementholder, the student received feedback in the form of a blinking LED, and the GUI projected the updated argument map on the wall. If a student wanted to more closely examine a piece of evidence, he or she could place the statement on the magnifying glass reader in the middle of the table, which would project the corresponding Web page on the screen.

The students constructed their arguments by arranging the statement-card TUIs, which were then projected on the display. Furthermore, placing a special speaker card on the desired statementholder triggered the GUI to display content relating to the specific section of the argument being presented. The projection would change from an overall argument map to a slide with bullet points for each statement (see figure 3c). These served as talking points for the student's presentation. The student could move the speaker card from statement-holder to statement-holder, literally following his or her line of argument.

The main issues with this prototype had to do with the RFID technology's performance and the system configuration. Although the RFID readers usually identified the statement cards placed on the statement-holders, the statements sometimes flickered on and off in the GUI. Furthermore, the individual statement-holders could read only six tags. The RFID's performance was sufficiently poor to distract the students' attention from the task of arranging their statements. Once again, introducing the technology derailed the lesson.

Another problem was configuration. Each statement the students identified from the Web pages had to be printed on a card and associated with an RFID taga laborious task. Also, the transition between the encomium, vituperation, and thesis exercises required some clumsy "scene shifting" as the researchers and teacher changed the printed labels on the statement-holder.

\section{Iteration 11: Final prototype}

To improve reliability, we equipped each argument square with two RFIDreading antennae. We also used "historicization" for the GUI, so that a tag that had already been read wasn't removed from the GUI display until it was absent from that argument square for five seconds. We also increased the number of statements the statementholder could hold.

To ease the burden of configuring statement cards, we developed software for a wireless-enabled tablet PC. The students selected text from the Web pages, and the software automatically printed the statements in business card format and assigned them RFID tags.

We also redesigned the statementholders to exploit tablet PCs as displays that could prompt the argument stages. The large projection screen continued to display the overall argument map, responding to the speaker tag and the items placed on the magnifying glass.
At the same time, we changed how we ran the trials. Because we wanted to design both the technology and the accompanying educational material, we had to iterate on the lesson plan. We decided that the preparatory worked required more time, so we altered the lesson to give students more time to read through the recommended pages, research their subject, and use the selection software to select relevant quotations.

Also, earlier trials involved students with mixed abilities—some were bright for their age and others had special educational needs. Evaluating the technology with mixed-ability students presented artificial challenges for analyzing the prototype's effectiveness-real classroom deployment would more likely be with groups of more similar ability. The facilitator addressed this in the final set of trials by having us work with a set of slightly older children (11 to 12 years old) who were all at the higher end of ability for their age group. Our CFD approach meant we had to not only engineer our classroom technology but also ensure the academic context around it was appropriate.

With this final iteration, the application finally began to "disappear." The changes in RFID-reading hardware, combined with the historicization software, let the students focus on arranging their statements. They had little or no difficulty understanding how to use the TUIs to arrange and structure their research. Discussion focused on deciding which statements should go in which argument stages and on filling in stages that had few or no statements. Without prompting, the students searched the Internet to look for pages that would plug the holes the TUI had revealed in their arguments. Furthermore, our prototype and lesson encouraged equal participation from all students, overcoming the girls' tendency to be verbally precocious. 
I terative design is particularly well suited for TUI design. Using lowtech prototypes lets you quickly and inexpensively test many of the more important aspects of TUI design-those surrounding integration with the physical context of use. You can also perform in-depth studies of single ideas and test several ideas that explore different aspects of the design space. Our initial prototypes invalidated our ideas about how TUIs might be useful. This alone revealed the value of iterative low-tech prototyping by letting us explore the application space before committing too much effort to software or hardware development.

Successful iterative prototyping using user-centered design involves tackling design issues in a wide area of user experience beyond the software and hardware's appearance and functionality. Prolonged periods of iterative design help the application designer understand the users, determine an application's best use, and identify a suitable use environment. Knowing how the technology fits into the larger social environment-in our case, the lessons-is what renders the technology invisible. We hope our experiences in shaping both the technology and the social context help others understand how to develop ubiquitous computing technology for a broad range of application spheres. $\mathbf{D}$

\section{ACKNOWLEDGMENTS}

We thank Chris Vernall for helping in creating the prototypes, Kate McCluskey for her initial ethnography, and Charlotte Lee and Paul Dourish for feedback on earlier drafts. European Union grant IST2001-34171 funded this research, but this article doesn't represent their opinions, nor are they responsible for the data.

\section{REFERENCES}

1. M. Weiser, "The Computer for the 21st Century," Scientific American, Sept. 1991, pp. 66-75.

2. J. Rode et al., "Curriculum Focused Design," Proc. 2003 Conf. Interaction

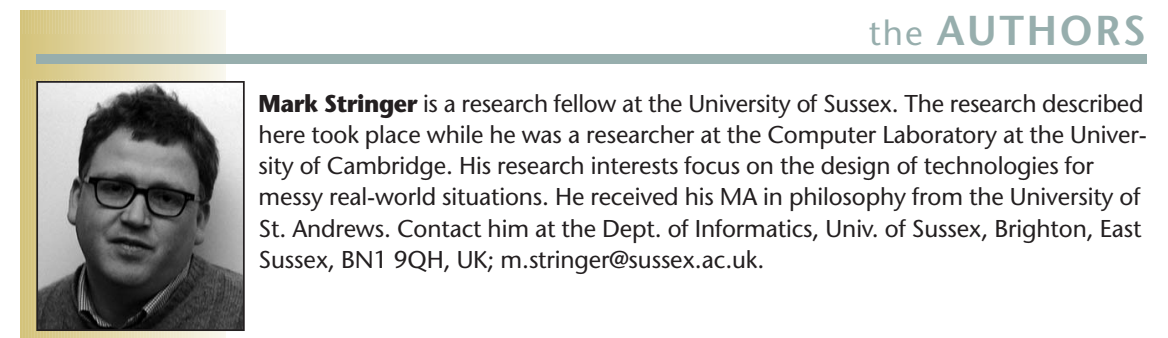

Jennifer A. Rode is a PhD student in informatics at the University of California, Irvine. She participated in this research while she was a research fellow at the University of Cambridge. She spent time at TiVo doing usability work and her research interests include ethnographic studies to inform both domestic ubiquitous computing applications and tangible interfaces for children. She received her MS in humancomputer interaction from Carnegie Mellon University. Contact her at the Dept. of Informatics, Univ. of California, Irvine, 444 Computer Science Bldg., Irvine, CA 92697-3425; jen@ics.uci.edu.

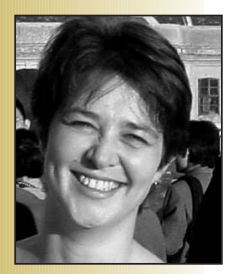

Eleanor F. Toye is a member of the User Research team at Framfab UK. Her research interests include studying how people adopt and use technology in their daily lives - particularly domestic technologies, educational technologies, and mobile phones. She received her PhD in experimental psychology from the University of Cambridge. Contact her at Framfab UK,1 Naoroji St., London, WC1X 0JD, UK; eleanor.toye@framfab.com.

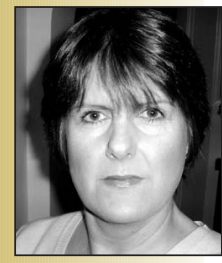

Amanda R. Simpson is a senior lecturer in mathematics education at the University of Central England. She received her MA in mathematics education from Warwick University and is conducting research for her $\mathrm{PhD}$, considering cognitive processes involved with children's learning and knowledge transfer in mathematics. Contact her at the Univ. of Central England, Attwood Bldg., Perry Barr, Birmingham, B42 2SU, UK; amanda.simpson@uce.ac.uk.

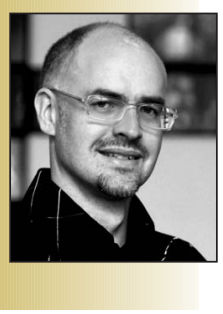

Design and Children (IDC 03), ACM Press, 2003, pp. 119-126.

3. A. Blackwell, "Cognitive Dimensions of Tangible Programming Languages," Proc. 1st Joint Conf. Empirical Assessment of Software Eng. and Psychology of Programming Interest Group (EASE and PPIG 03), 2003, pp. 391-405.

4. C. Crook, "Children as Computer Users," Computer Education, vol. 30, nos. 3-4, 1988, pp. 237-247.

5. L. Hanna et al., "The Role of Usability Research in Designing Children's Computer Products," The Design of Children's Technology, Morgan Kaufmann, 1999, pp. 3-26.

6. K. Inkpen et al., "Playing Together Beats Playing Apart, Especially for Girls," Proc. 1st Int'l Conf. Computer Support for Collaborative Learning (CSCL 1995),
Lawrence Erlbaum, 1995, pp. 177-181.

7. E. Soloway et al., "Learning Theory in Practice: Case Studies of Learner-Centered Design," Proc. SIGCHI Human Factors Computing Systems (CHI 96), ACM Press, 1996, pp. 189-196.

8. A. Druin, “Cooperative Inquiry: Developing New Technologies for Children with Children," Proc. SIGCHI Human Factors in Computing Systems (CHI 99), ACM Press, 1996, pp. 592-599.

9. A.J. Sellen and R.H.R. Harper, The Myth of the Paperless Office, MIT Press, 2003.

10. Visualizing Argumentation: Software Tools for Collaborative and Educational SenseMaking, P. Kirschner, S. Buckingham Shum, and C. Carr, eds., Springer, 2002.

11. E.P.J. Corbett and R.J. Connors, Classical Rhetoric for the Modern Student, Oxford Univ. Press, 1998. 